\title{
INSECT SEX CHROMOSOMES: IX. X CHROMOSOME LINKAGE OF GLUCOSE 6-PHOSPHATE DEHYDROGENASE (G-6-PD) LOCUS IN GRYLLOTALPA FOSSOR (ORTHOPTERA)
}

\author{
S. R. V. RAO AND MEENAKSHI BHATTACHARYA \\ Department of Zoology, University of Delhi, Delhi-110007, India
}

Received 10.i.83

\section{SUMMARY}

\begin{abstract}
Electrophoretic analysis of G-6-PD from single individuals of Gryllotalpa fossor from a wild population indicated that this locus is $\mathrm{X}$-linked and the polymorphic forms could be identified by the variations in their mobility (fast and slow). The presence of both the bands in single female individuals supports our views (Rao and Arora 1979; Arora and Rao 1980) that there is random inactivation of one of the two Xs in the somatic cells. Further, it is postulated that the linkage of G-6-PD with the $X$ chromosome in the male heterogametic organisms is due to selection pressure.
\end{abstract}

\section{INTRODUCTION}

Our recent studies on Gryllotalpa fossor have established the occurrence of a differential regulation mechanism of the $X$ chromosome by inactivation (facultative heterochromatisation) of one of the two Xs in the female somatic cells (Rao and Arora, 1979; Arora and Rao, 1980). In such a case, the female would be expected to produce a mosaic of $X$ chromosome activity (gene products). In pursuance of these studies, we have attempted to look for certain X-linked gene markers known to show variation in their electrophoretic mobility. One such a marker is the enzyme G-6-PD, which happens to be X-linked and to show variation in a variety of mammals including man (Ohno, 1967), Drosophila (Diptera; Young et al., 1964) and Tribolium, (Coleoptera; Dawson and Hollingsworth, 1982). Thus, it is reasonable to suppose that the G-6-PD locus would be X-linked and show polymorphism in Gryllotalpa too. A search for such a polymorphism was undertaken by examining the homogenates of single individuals and we report our results here.

\section{Materials AND methods}

Male and female Gryllotalpa of the fourth instar were utilised for the experiment. They were separately dissected in cold isotonic saline $(0.75 \mathrm{per}$ cent $\mathrm{NaCl}$ ). Barring the hepatic caeca, the alimentary canal was discarded and the rest of the animal, along with the hepatic caeca, was homogenised thoroughly in a glass homogeniser. The homogenising buffer was comprised of $0.1 \mathrm{M}$ Tris $\mathrm{HCl} p \mathrm{H} 6.7$ to which NADP (Sigma) was added to a final concentration of $2 \mathrm{mg} / \mathrm{ml}$. All procedures from dissection onwards were carried out at $0-4^{\circ} \mathrm{C}$. The homogenate was centrifuged at $3000 \mathrm{rpm}(1500 \mathrm{Xg})$ for fifteen minutes at $0-4^{\circ} \mathrm{C}$ and the clear supernatants were utilised for electrophoresis. 
Electrophoresis was carried out in 7 per cent polyacrylamide disc gels according to the technique of Davis (1964) using a discontinuous buffer system. Samples were mixed with 40 per cent sucrose and Bromophenol blue as marker dye and carefully loaded on to the gels. Runs were carried out at $4^{\circ} \mathrm{C}$ for about $2 \frac{1}{2}-3 \mathrm{~h}$ at the rate of $3 \mathrm{~mA} /$ tube.

The gels were stained at $37^{\circ} \mathrm{C}$ for $10-30 \mathrm{~min}$ in the dark in a staining mixture containing $20 \mathrm{ml}$ Tris $\mathrm{HCl} p \mathrm{H} \mathrm{7.4,12.5} \mathrm{mg} \mathrm{of} \mathrm{D-glucose-6-phos-}$ phate (Sigma), $3.25 \mathrm{mg}$ of Nitro blue tetrazolium, $2 \mathrm{mg}$ of phenazine methosulphate and $2 \mathrm{ml}$ of $0.01 \mathrm{M} \mathrm{MgCl}_{2}$. Stained gels were fixed and then preserved in 7 per cent acetic acid and photographed immediately on ORWO 125 ASA film. Out of 70 animals only 61 individuals were analysable for electrophoretic bands (table 1).

TABLE 1

Phenotype frequencies for G-6-pd in Gryllotalpa fossor

\begin{tabular}{|c|c|c|c|c|c|c|c|}
\hline & \multicolumn{2}{|c|}{ Fast } & \multicolumn{2}{|c|}{ Fast/Slow } & \multicolumn{2}{|c|}{ Slow } & \multirow[b]{2}{*}{ Tota } \\
\hline & Obs. & Exp. & Obs. & Exp. & Obs. & Exp. & \\
\hline \multirow{3}{*}{$\begin{array}{l}\text { Male } \\
\text { Female }\end{array}$} & 22 & $(19 \cdot 82)$ & 0 & $(4 \cdot 57)$ & 9 & $(6.61)$ & 31 \\
\hline & 17 & $(19 \cdot 18)$ & 9 & $(4.43)$ & 4 & $(6 \cdot 39)$ & 30 \\
\hline & 39 & & 9 & & 13 & & 61 \\
\hline
\end{tabular}

$\chi^{2}=11 \cdot 530053 . \mathrm{df}=2$. Significant at 5 per cent level.

\section{RESUlts AND GENERAL CONSIDERATIONS}

At the outset, it should be mentioned that there is no information on the genetics of Gryllotalpa. This is due, apparently, to the fact that the life cycle in Gryllotalpa fossor is highly extended as evidenced by its congeneric species G. gryllotalpa where it occupies about two years (Camenzind and Nicklas, 1968). Hence our approach was to screen single individuals from the population. According to the X-chromosome linkage model, and presuming that there are two variants for this locus (fast and slow), the males from the population should show only a single electrophoretic band (fast or slow) whereas the female would show the following phenotypes, either fast or slow bands (homozygous) or both the bands (heterozygous). As expected, our results corroborate the above expectations (plate 1). An analysis of 61 individuals from the Delhi population indicates (table 1) that out, of 31 males, 22 showed a fast band and 9 showed a slow band. None of the males showed both the bands. Of 30 females analysed, 17 showed a fast band (homozygous), 4 showed a slow band (also homozygous) and 9 showed both fast and slow bands (heterozygous). Apparently, in these heterozygotes there are two different populations of cells coding for these phenotypes.

In order to establish the nature of linkage (autosomal or sex chromosomal) a $2 \times 3$ contingency test was carried out on the assumption that the relative proportions of the three phenotypes are the same in both sexes and that natural selection (if any) acts on this locus in much the same way in either sex. Accordingly, the expected numbers are calculated (table 1). The $\chi^{2}$ value is 11.530053 and for $2 \mathrm{df}$ at 5 per cent level it is significant. Thus, 


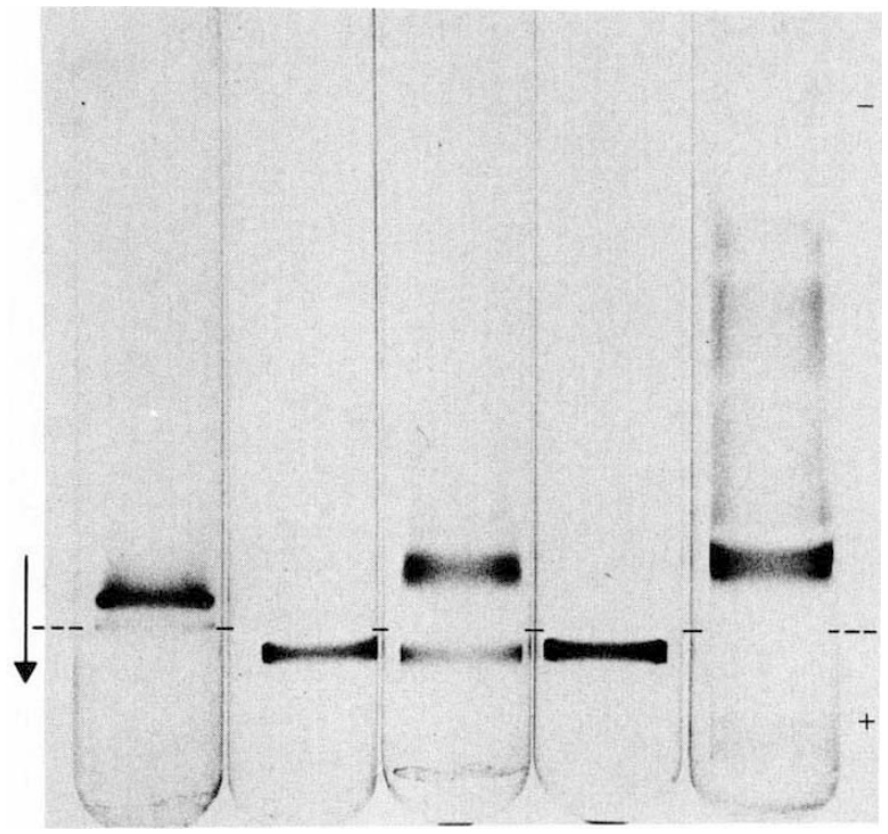

Plate 1. G-6-PD phenotypes of single male and female Gryllotalpa. Arrow indicates the direction of migration. The dotted line points to the marker dye. 
autosomal linkage is ruled out and the $\mathrm{X}$-linkage is in evidence. The absence of any heterozygous males is in order (table 1). This is what is expected of X-chromosome linkage model for organisms with male heterogamety.

In order to test the hypothesis of sex linkage the nearest one can get to a reliable test is to try to test the adequacy of the model designating $F$ for fast, $\mathrm{S}$ for slow.

\begin{tabular}{lccccc} 
& \multicolumn{3}{c}{ Female } & \multicolumn{3}{c}{ Male } \\
& $\mathrm{F}$ & $\mathrm{F} / \mathrm{S}$ & $\mathrm{S}$ & $\mathrm{F}$ & $\mathrm{S}$ \\
Exptd. proportions & $P$ & $2 Q$ & $R$ & $P+Q$ & $Q+R$ \\
Obs. numbers & $a$ & $b$ & $c$ & $d$ & $e$
\end{tabular}

To do this the following simultaneous equations obtained by standard maximum likelihood procedures are solved:

$$
\begin{aligned}
-\frac{a}{p}+\frac{b}{2 Q}-\frac{d}{2(P+Q)}+\frac{e}{2(Q+R)} & =0 \\
-\frac{c}{R}+\frac{b}{2 Q}+\frac{d}{2(P+Q)}-\frac{e}{2(Q+R)} & =0 \\
P+2 Q+R & =1 .
\end{aligned}
$$

This model assumes no selection and also makes no assumptions about the mating systems. The observed results (table 1 ) then yield the estimates $\hat{P}=0.563426, \hat{Q}=0.150649, \hat{R}=0.135276$ giving

$\begin{array}{llllll}\text { Observed } & 17 & 9 & 4 & 22 & 9 \\ \text { Expected } & 16.9028 & 9 \cdot 0389 & 4 \cdot 0583 & 22 \cdot 1363 & 8 \cdot 8637\end{array}$

The $\chi^{2}=0 \cdot 1091762$ and, at $1 \mathrm{df}$ (since $2 \mathrm{df}$ have already been used up in estimating $P, Q$, and $R$, two of which are independent), the value is insignificant even at the 1 per cent level. Thus the fit is excellent and establishes beyond doubt that the G-6-PD locus in Gryllotalpa is indeed $\mathrm{X}$-linked and the alleles are co-dominant. Additionally, the fact that some females in the population show both fast and slow G-6-PD phenotypes, substantiates that the $\mathrm{X}$-chromosome inactivation mechanism is operative in Gryllotalpa (Rao and Arora, 1979; Arora and Rao, 1980).

The discovery that the G-6-PD locus in the orthopteran, Gryllotalpa, is also X-linked leads one to question whether this locus is not X-linked in all organisms with male heterogamety. The X-chromosome in Gryllotalpa is metacentric and comprises 18 per cent of the haploid genome (Rao and Arora, 1979). This would mean that in Gryllotalpa the probability of G-6-PD locus being found on the $\mathrm{X}$ chromosome is about one in five. A perusal of the literature shows that, apart from birds, wherever G-6-PD has been demonstrated i.e., in various mammals including man (Ohno 1967), in Drosophila (Young et al., 1964), Tribolium (Dawson and Hollingsworth, 1982) and Gryllotalpa (this report) it is indeed X-linked. The question would then be whether its location on the X-chromosome of these widely divergent organisms is fortuitous or results from some selection pressure which maintains such a relationship (Young et al., 1964). Interestingly, all the organisms where G-6-PD has been shown to be X-linked genes, show male 
heterogamety. The one exception, birds, where G-6-PD is not X-linked and where no dosage compensation occurs (Ohno, 1967), show female heterogamety.

For the purpose of speculation, and to update the calculation first made by Young $e t$ al. (1964), we have taken the size of the metaphase X chromosome into account to consider the probability of the G-6-PD locus being present on the $\mathrm{X}$-chromosome. We propose to designate the proportion of the $\mathrm{X}$ in the genome of mammals (including man), Drosophila, Tribolium, Gryllotalpa and birds respectively, as $P_{1}, P_{2}, P_{3}, P_{4}$ and $P_{5}$. The respective size value of the $X$ chromosome per haploid set for mammals is about 0.06 (Ohno, 1967), Drosophila, about 0.2 (Young et al., 1964), Tribolium about 0.1 (Dawson and Hollingsworth, 1982), Gryllotalpa about 0.2 (Rao and Arora, 1979) and for birds about 0.1 (Ohno, 1967). The probability $(P)$ of G-6-PD locus being X-linked in any four of the five groups can be calculated using the following formula (adapted from Dawson and Hollingsworth, 1982):

$$
\begin{aligned}
P= & P_{1} P_{2} P_{3} P_{4}\left(1-P_{5}\right)+P_{1} P_{2} P_{3}\left(1-P_{4}\right) P_{5}+P_{1} P_{2}\left(1-P_{3}\right) P_{4} P_{5} \\
& +P_{1}\left(1-P_{2}\right) P_{3} P_{4} P_{5}+\left(1-P_{1}\right) P_{2} P_{3} P_{4} P_{5} .
\end{aligned}
$$

The $P$ value is found to be 0.001 , that is approximately 1 in 1000 . Though the probability of the G-6-PD locus being on the $\mathrm{X}$ chromosome as calculated in terms of the size of the $X$ is very low, the fact that this locus is indeed X-linked in all four evolutionarily divergent species with male heterogamety strongly suggests a non-random association of the locus with the $\mathrm{X}$-chromosome. It appears that selection pressure has indeed seemed to maintain this locus on the X-chromosome.

Acknowledgments. We thank Professor Bernard John, Department of Population Biology, Ressearch School of Biological Sciences, Australian National University, Canberra City, Australia, for criticism and helpful suggestions. This work was supported by a research fellowship from the University Grants Commision, Delhi (M.B.) and by the research grant (No. HCS/DST/658/78) from the Department of Science \& Technology, Government of India, Delhi (S.R.V.R.).

\section{REFERENCES}

ARORA, P. AND RAO, S. R. V. 1980. Insect sex chromosomes. V. ${ }^{3} \mathrm{H}$-uridine induced aberrations in the $\mathrm{X}$ chromosomes of tetraploid spermatogonia from Gryllotalpa fossor. Chromosoma, (Berl.), 77, 373-378.

CAMENZIND, R. AND NICKLAS, R. B. 1968. The non-random chromosome segregation in spermatocytes of Gryllotalpa hexadactyla. A micromanipulation analysis. Chromosoma, (Berl.), 24, 324-335.

DAVIS, B. J. 1964. Disc electrophoresis. II. Methods and application to human serum proteins. Ann. N.Y. Acad. Sci., 121, 404-421.

DAWSON, P. S. AND HOLLINGSWORTH, N. N. 1982. Sex linkage of the glucose-6-phosphate dehydrogenase locus in the flour beetle Tribolium castaneum. Can. J. Genet. Cytol., 24, 267-271.

oHNO, s. 1967. Sex Chromosomes and Sex-linked Genes. Springer-Verlag, Berlin-HeidelbergNew York.

RAO, S. R. V. AND ARORA, P. 1979. Insect sex chromosomes. III. Differential susceptibility of homologous $\mathrm{X}$ chromosomes of Gryllotalpa fossor to ${ }^{3} \mathrm{H}$-Urd-induced aberrations. Chromosoma, (Berl.), 74, 241-252.

YOUNG, W. J., PORTER, J. E. AND CHILDS, B. 1964. Glucose-6-phosphate dehydrogenase in Drosophila: X-linked electrophoretic variants. Science, 143, 140-141. 